\title{
Privacy-Preserving Data Mining: A Game-Theoretic Approach
}

\author{
Atsuko Miyaji and Mohammad Shahriar Rahman \\ School of Information Science, Japan Advanced Institute of Science and Technology \\ 1-1 Asahidai, Nomi, Ishikawa, Japan 923-1292 \\ \{miyaji,mohammad\}@jaist.ac.jp
}

\begin{abstract}
Privacy-preserving data mining has been an active research area in recent years due to privacy concerns in many distributed data mining settings. Protocols for privacy-preserving data mining have considered semi-honest, malicious, and covert adversarial models in cryptographic settings, whereby an adversary is assumed to follow, arbitrarily deviate from the protocol, or behaving somewhere in between these two, respectively. Semi-honest model provides weak security requiring small amount of computation, on the other hand, malicious and covert models provide strong security requiring expensive computations like homomorphic encryptions. However, game theory allows us to design protocols where parties are neither honest nor malicious but are instead viewed as rational and are assumed (only) to act in their own self-interest. In this paper, we build efficient and secure set-intersection protocol in gametheoretic setting using cryptographic primitives. Our construction avoids the use of expensive tools like homomorphic encryption and oblivious transfer. We also show that our protocol satisfies computational versions of strict Nash equilibrium and stability with respect to trembles.
\end{abstract}

Keywords: Privacy-preserving data mining, Set-intersection, Game theory, Computational strict Nash equilibrium, Stability with respect to trembles.

\section{Introduction}

A key utility of large databases today is scientific or economic research. Despite the potential gain, this is often not possible due to the confidentiality issues which arise, leading to concerns over privacy infringement while performing the data mining operations. The need for privacy is sometimes due to law (e.g., for medical databases) or can be motivated by business interests. To address the privacy problem, several privacy-preserving data mining protocols using cryptographic techniques have been suggested. Depending on the adversarial behavior assumptions, those protocols use different models. Classically, two main categories of adversaries have been considered:

Semi-honest adversaries: Following Goldreich's definition [11], protocols secure in the presence of semi-honest adversaries (or honest-but-curious) assume

Y. Li (Ed.): Data and Applications Security and Privacy XXV, LNCS 6818, pp. 186-200, 2011.

(C) IFIP International Federation for Information Processing 2011 
that parties faithfully follow all protocol specifications and do not misrepresent any information related to their inputs, e.g., set size and content. However, during or after protocol execution, any party might (passively) attempt to infer additional information about the other party's input. This model is formalized by requiring that each party does not learn more information that it would in an ideal implementation relying on a trusted third party (TTP).

Malicious adversaries: Security in the presence of malicious parties allows arbitrary deviations from the protocol. In general, however, it does not prevent parties from refusing to participate in the protocol, modifying their private input sets, or prematurely aborting the protocol. Security in the malicious model is achieved if the adversary (interacting in the real protocol, without the TTP) can learn no more information than it could in the ideal scenario.

A new type of adversarial model, named covert adversary, has been proposed recently by Aumann et al. [3].

Covert Adversaries: These adversaries are somewhere in between the semihonest and malicious models. In many real-world settings, parties are willing to actively cheat (not semi-honest), but only if they are not caught (not arbitrarily malicious). Covert adversarial behavior accurately models many real-world situations. It explicitly models the probability of catching adversarial behavior; a probability that can be tuned to the specific circumstances of the problem. In particular, it is not assumed that adversaries are only willing to risk being caught with negligible probability, but rather allow for much higher probabilities.

In the above models, a secure protocol emulates (in its real execution) the ideal execution that includes a TTP. This notion is formulated by requiring the existence of adversaries in the ideal execution model that can simulate adversarial behavior in the real execution model. In other words, the implicit assumption in the original formulation of the problem is that each party is either honest or corrupt, and honest parties are all willing to cooperate when reconstruction of the secret is desired. However, the assumption of semi-honest behavior may be unrealistic in some settings. In such cases, participating parties may prefer to use a protocol that is secure against malicious behavior. It is clear that the protocols secure in the malicious model offer more security. Regarding malicious adversaries, it has been shown that, under suitable cryptographic assumptions, any multi-party probabilistic polynomial time functionality (PPT) can be securely computed for any number of malicious corrupted parties. However, these are not efficient enough to be used in practice. Most of these constructions use general zero-knowledge proofs for fully malicious multi-party computation (MPC) protocols. These zeroknowledge compilers lead to rather inefficient constructions [31. In typical cryptographic MPC protocols, parties are allowed to abort when they can find some malicious behavior from other parties. This means that the parties have to start the protocol from the scratch which is undesirable for operations on huge data sets.

Since the work of Halpern and Teague [14, protocols for some cryptographic tasks (e.g., secret sharing, multi-party computation) have begun to be reevaluated in a game-theoretic light (see 7/20, for an overview of work in this 
direction). In this setting, parties are neither honest nor corrupt but are instead viewed as rational and are assumed (only) to act in their own self-interest. This feature is particularly interesting for data mining operations where huge collection of data is used, since parties will not deviate (i.e., abort) as there is no incentive to do so. In many real-world settings, parties are willing to actively deviate/cheat, but only if they are not caught. This is the case in many business, financial, political and diplomatic settings, where honest behavior cannot be assumed, but where the companies, institutions and individuals involved cannot afford the embarrassment, loss of reputation, and negative press associated with being caught cheating, hence having smaller incentive.

In data mining area, private set-intersection and set-union protocols allow two parties interact on their respective input sets. These protocols address several realistic privacy issues. Typical application examples include:

1. Business Interest: Companies may want to decide whether to make a business alliance by the percentage of customers shared among them, without publishing their customer databases including the shared customers among them. This can be treated as an intersection cardinality problem. As another example, to determine which customers appear on a do-not-receive-advertisements list, a store must perform a set-intersection operation between its private customer list and the producers list.

2. Aviation Security: The Department of Homeland Security (DHS) of the U.S. needs to check whether any passenger on each flight from/to the United States must be denied boarding, based on some passenger watch list. For this purpose, airlines submit their entire list of passengers to DHS, together with other sensitive information, such as credit card numbers. This poses liability issues with regard to innocent passengers' data and concerns about potential data losses. In practice, information only related to the passengers on the list should obtained by DHS without disclosing any information to the airlines.

3. Healthcare: Insurance companies often need to obtain information about their insured patients from other parties, such as other insurance carriers or hospitals. The insurance carriers cannot disclose the identity of inquired patients, whereas, the hospitals cannot provide any information on other patients.

\section{$1.1 \quad$ Related Work}

Cryptographic techniques have been used to design many different distributed privacy-preserving data mining algorithms. In general, there are two types of assumptions on data distribution: vertical and horizontal partitioning. In the case of horizontally partitioned data, different sites collect the same set of information about different entities. For example, different credit card companies may collect credit card transactions of different individuals. Secure distributed protocols have been developed for horizontally partitioned data for mining decision trees [25], k-means clustering [24], k-nn classifiers [18]. In the case of vertically partitioned data, it is assumed that different sites collect information about the same set of entities but they collect different feature sets. For example, both a university and a hospital may collect information about a student. Again, secure 
protocols for the vertically partitioned case have been developed for mining association rules [35], and k-means clusters [1634]. All of those previous protocols claimed to be secure only in the semi-honest model. In 919, authors present two-party secure protocols in the malicious model for data mining. They follow the generic malicious model definitions from the cryptographic literature, and also focus on the security issues in the malicious model, and provide the malicious versions of the subprotocols commonly used in previous privacy-preserving data mining algorithms. Assuming that at least one party behaves in semi-honest model, they use threshold homomorphic encryption for malicious adversaries presented by Cramer et al. [5]. Recently, Miyaji et al. presented a new adversarial model named covert adversaries [31] for performing data mining algorithms. They show that protocols under covert adversarial model behave in between semi-honest and malicious models. Oblivious transfer (OT) and homomorphic encryption have been used as the building blocks in 31. Since homomorphic encryption is considered too expensive [27] and oblivious transfer is often the most expensive part of cryptographic protocols [26], the protocols proposed in malicious and covert adversarial models are not very practical for operations on large data items. Game theory and data mining, in general, have been combined in 17.32 for constructing various data mining algorithms. Rational adversaries have also been considered in privacy-preserving set operations [36 2 . These protocols consider Nash equilibrium to analyze the rational behavior of the participating entities. As discussed by Kol and Naor in [23], using Nash equilibrium is not suitable in many cases, since many bad strategies are not ruled out by it. Instead, they suggest the stronger notion of strict Nash equilibrium in the information-theoretic setting, in which every player's strategy is a strict best response. Due to the restrictive nature of this notion, it is regarded as a sufficient condition and not as a necessary one. As in all of cryptography, computational relaxations are meaningful and should be considered; doing so allows us to get around the limitations of the information-theoretic setting. So, analyzing set operations from the viewpoint of computational strict Nash equilibrium is interesting, since it gives a more realistic results. There have been several works on game theory based MPC/secret sharing schemes 11422 2910 3315. But 1433 require the continual involvement of the dealer even after the initial shares have been distributed or assume that sufficiently many parties behave honestly during the computation phase. Some schemes [122 29] rely on multiple invocations of protocols. Other work 15 relies on physical assumptions such as secure envelopes and ballot boxes. 10 proposed efficient protocols for rational secret sharing. But secret sharing schemes cannot be directly used for our purpose since they require the existence of TTP and their set up is different.

\subsection{Our Contribution}

In this work, we build two-party secure set-intersection protocol in gametheoretic setting using cryptographic primitives. It is assumed that parties are neither honest nor corrupt but are instead rational and are assumed to act only in their own self-interest. Our construction avoids the use of expensive tools like 
homomorphic encryption and oblivious transfer. We have used verifiable random functions as the underlying cryptographic primitive which is simple and efficient. It is also possible to use our protocol for computing set-union operations. We also show that our protocol satisfies computational versions of strict Nash equilibrium and stability with respect to trembles, defined by Fuchsbauer et al. [10].

Organization of the paper: The remainder of the paper is organized as follows: Section 2 presents the background and preliminaries. Section 3 describes the protocol model. Section 4 includes protocol construction. In Section 5, we analyze the protocol formally. We give some concluding remarks in Section 6 .

\section{Background and Preliminary}

\subsection{Cryptographic Considerations in Game Theory}

Achieving a secure protocol is the objective in the cryptographic setting. Eliminating the trusted party is one of the main tasks while maintaining the privacy. On the other hand, in game theory, some particular equilibrium is defined to achieve stability. The existence of the trusted party/mediator is a parameter setting resulting in a more desirable, but harder to implement equilibrium concept for rational behaviors. Thus, privacy is a goal in the cryptographic setting while in the game theory setting it is a means to an end.

Games are treated in a modified way with a differently defined equilibrium notions in a cryptographic setting with. Katz, in [20], gives some examples of how this might be done for the specific case of parties running a protocol in the cryptographic setting. A security parameter $n$ is introduced which is provided to all parties at the beginning of the game. The action of a player $P_{j}$ now corresponds to running an interactive Turing Machine (TM) $T_{j}$. The $T_{j}$ takes the current state and messages received from the other party as the input, and outputs message of player $P_{j}$ along with updated state. The message $m_{j}$ is sent to the other party. In a computational sense, it is required that $T_{j}$ runs in PPT meaning that the function is computed in time polynomial in $n . T_{j}$ is thus allowed to run for an unbounded number of rounds and, it can be added that the expected number of rounds is also polynomial for which $T_{j}$ runs. The security parameter $n$ is given as input to the utility functions. Utility functions map transcripts of a protocol execution to the reals that can be computed in time polynomial in $n$. Let $\Delta$ be a computational game in which the actions of each player correspond to the PPT TMs. Also, the utilities of each player are computed in time polynomial in $n$. Thus, mixed strategies are no longer needed to be considered, since a polynomial time mixed strategy corresponds to a pure strategy (since pure strategies correspond to randomized TMs) [20]. The parties are not assumed to be curious in negligible changes in their utilities, and this is an important difference between the cryptographic setting and the setting that has been considered here. 


\subsection{Definitions}

In this section, we will state the definitions of computational strict Nash equilibrium and computational strict Nash equilibrium w.r.t. trembles introduced in [10. A protocol is in Nash equilibrium if no deviations are advantageous; it is in strict Nash equilibrium if all deviations are disadvantageous. In other words, there is no incentive to deviate in the case of a Nash equilibrium whereas there is an incentive not to deviate for a strict Nash equilibrium. Another advantage of strict Nash is that protocols satisfying this notion inhibit subliminal communication. A party who tries to use protocol messages as a covert channel has the risks to lose utility if there is any reasonable probability that the other player is following the protocol, since any detectable deviation by a party from the protocol results in lower utility while the other party follows the protocol. The computational version of strict Nash equilibrium is intuitively close to strict Nash considering the computational limitations. Moreover, our protocol satisfies a strong condition that each party can send a unique legal message that at every point in the protocol. Our protocol thus rules out subliminal communication in a strong sense. We denote the security parameter by $n$. A function $\epsilon$ is negligible if for all $c>0$ there is a $n_{c}>0$ such that $\epsilon(n)<1 / n^{c}$ for all $n>n_{c}$; let negl denote a generic negligible function. We say $\epsilon$ is noticeable if there exist $c, n_{c}$ such that $\epsilon(n)>1 / n^{c}$ for all $n>n_{c}$.

We consider the strategies in our work as the PPT interactive Turing machines. Given a vector of strategies $\boldsymbol{\sigma}$ for two parties in the computation phase, let $u_{j}(\boldsymbol{\sigma})$ denote the expected utility of $P_{j}$, where the expected utility is a function of the security parameter $n$. This expectation is taken over the randomness of the players' strategies. Following the standard game-theoretic notation, $\left(\sigma_{j}^{\prime}, \boldsymbol{\sigma}_{-j}\right)$ denotes the strategy vector $\boldsymbol{\sigma}$ with $P_{j}$ 's strategy changed to $\sigma_{j}^{\prime}$.

Definition 1. $\Pi$ induces a computational Nash equilibrium if for any PPT strategy $\sigma_{1}^{\prime}$ of $P_{1}$ we have $u_{1}\left(\sigma_{1}^{\prime}, \sigma_{2}\right) \leq u_{1}\left(\sigma_{1}, \sigma_{2}\right)+$ negl $(n)$, and similarly for $P_{2}$.

The computational notion of stability with respect to trembles models players' uncertainty about other parties' behavior, and guarantees that even if a party $P_{i}$ believes that other parties might play some arbitrary strategy with small probability $\delta$ (but follow the protocol with probability $1-\delta$ ), there is still no better strategy for $P_{i}$ than to follow the protocol. The following definition is stated for the case of a deviating $P_{1}$ (definition for a deviating $P_{2}$ is analogous). Let $P_{1}$ and $P_{2}$ interact, following $\sigma_{1}$ and $\sigma_{2}$, respectively. Let mes denote the messages sent by $P_{1}$, but not including any messages sent by $P_{1}$ after it writes to its (write-once) output tape. Then view $w_{2}^{\Pi}$ includes the information given by the trusted party to $P_{2}$, the random coins of $P_{2}$, and the (partial) transcript mes. We fix a strategy $\gamma_{1}$ and an algorithm $A$. Now, let $P_{1}$ and $P_{2}$ interact, following $\gamma_{1}$ and $\sigma_{2}$, respectively. Given the entire view of $P_{1}$, algorithm $A$ outputs an arbitrary part $m e s^{\prime}$ of mes. Then view $w_{2}^{A, \gamma_{1}}$ includes the information given by the trusted party to $P_{2}$, the random coins of $P_{2}$, and the (partial) transcript $m e s^{\prime}$. 
Definition 2. Strategy $\gamma_{1}$ yields equivalent play with respect to $\Pi$, denoted $\gamma_{1} \approx$ $\Pi$, if there exists a PPT algorithm $A$ such that for all PPT distinguishers $D$

$\left|\operatorname{Pr}\left[D\left(1^{n}, \operatorname{view}_{2}^{A, \gamma_{1}}\right)=1\right]-\operatorname{Pr}\left[D\left(1^{n}, \operatorname{view}_{2}^{\Pi}\right)=1\right]\right| \leq \operatorname{negl}(n)$

Definition 3. $\Pi$ induces a computational strict Nash equilibrium if

1. $\Pi$ induces a computational Nash equilibrium;

2. For any PPT strategy $\sigma_{1}^{\prime} \not \approx \Pi$, there is a $c>0$ such that $u_{1}\left(\sigma_{1}, \sigma_{2}\right) \leq$ $u_{1}\left(\sigma_{1}^{\prime}, \sigma_{2}\right)+1 / n^{c}$ for infinitely many values of $n$.

In stability with respect to trembles, we say that $\gamma_{i}$ is $\delta$-close to $\sigma_{j}$ if with probability $1-\delta$ party $P_{j}$ plays $\sigma_{j}$, while with probability $\delta$ it follows an arbitrary PPT strategy $\sigma_{j}^{\prime}$. In fact, a pair of strategies $\left(\sigma_{1}, \sigma_{2}\right)$ is stable with respect to trembles if $\sigma_{1}$ (resp., $\sigma_{2}$ ) remains the best response even if the other party plays a strategy other than $\sigma_{2}$ (resp., $\sigma_{1}$ ) with some small (but noticeable) probability $\delta$. The fact that the prescribed strategies are in Nash equilibrium ensures that any (polynomial-time) local computation performed by either party is of no benefit as long as the other party follows the protocol. Stated differently, even if a party $P_{j}$ believes that the other party might play a different strategy with some small probability $\delta$, there is still no better strategy for $P_{j}$ than to outwardly follow the protocol.

Definition 4. $\Pi$ induces a computational strict Nash equilibrium that is stable with respect to trembles if

1. $\Pi$ induces a computational Nash equilibrium;

2. There is a noticeable function $\delta$ such that for any PPT strategy $\gamma_{2}$ that is $\delta$-close to $\sigma_{2}$, and any PPT strategy $\gamma_{1}$, there exists a PPT strategy $\sigma_{1}^{\prime} \approx \Pi$ such that $u_{1}\left(\gamma_{1}, \gamma_{2}\right) \leq u_{1}\left(\sigma_{1}^{\prime}, \gamma_{2}\right)+\operatorname{negl}(n)$

Verifiable Random Functions (VRFs): A VRF is a keyed function whose output is random-looking but can still be verified as correct, given an associated proof. The notion was introduced by Micali et al. [30, and various efficient constructions in the standard model are known 6 6 28. It has been shown in 28 that efficient VRFs can be constructed without relying on zero-knowledge proof $\left\{\right.$. A verifiable random function (VRF) with range $R=\left\{R_{n}\right\}$ is a tuple of PPT algorithms (Gen, Eval, Prove, Verify) such that: $G\left(1^{n}\right)$ generates the key pair $(p k, s k)$. $\operatorname{Eval}_{s k}(x)$ computes the value $y=F_{p k}(x) ; \operatorname{Prove}_{s k}(x)$ computes the proof $z$ that $y=F_{p k}(x)$; and $V$ erif $y_{p k}(x, y, z)$ verifies that $y=F_{p k}(x)$ using the proof $z$. For such a VRF, the following hold:

Correctness: For all $n$, the algorithm $E_{v a l}$ sk maps $n$-bit input to a set $R_{n}$. Furthermore, for any $x \in\{0,1\}^{n}$ we have $\operatorname{Verify}_{p k}\left(x, \operatorname{Eval}_{s k}(x)\right.$, Prove $_{s k}$ $(x))=1$.

Verifiability: For all $(p k, s k)$ output by $\operatorname{Gen}\left(1^{n}\right)$, there does not exist a tuple $\left(x, y, y^{\prime}, z, z^{\prime}\right)$ with $y \neq y^{\prime}$ and Verif $y_{p k}(x, y, z)=1=\operatorname{Verif} y_{p k}\left(x, y^{\prime}, z^{\prime}\right)$.

\footnotetext{
${ }^{1}$ The VRF gives us computational security. However, it is also possible to design our protocol with information-theoretic security using information-theoretically secure MACs. The details will appear in the full version.
} 
Unique proofs: For all $(p k, s k)$ output by $\operatorname{Gen}\left(1^{n}\right)$, there does not exist a tuple $\left(x, y, z, z^{\prime}\right)$ with $z \neq z^{\prime}$ and $\operatorname{Verif} y_{p k}(x, y, z)=1=\operatorname{Verif} y_{p k}\left(x, y, z^{\prime}\right)$.

Pseudorandomness: Let $\mathcal{A}$ be a PPT adversary in the following game:

1. Generate $(p k, s k) \leftarrow G e n\left(1^{n}\right)$ and give $p k$ to $\mathcal{A}$. $\mathcal{A}$ queries a sequence of strings $x_{1}, \ldots x_{l} \in\{0,1\}^{n}$ and is given $y_{i}=\operatorname{Eval}_{s k}\left(x_{i}\right)$ and $z_{i}=\operatorname{Prove}_{s k}\left(x_{i}\right)$ in response.

2. $\mathcal{A}$ outputs a string $x \in\{0,1\}^{n}$ s.t. $x \notin\left\{x_{1}, \ldots x_{l}\right\} \in\{0,1\}^{n}$.

3. $\mathcal{A}$ chooses a random $b \in\{0,1\}$. If $b=0$ then $\mathcal{A}$ is given $y=\operatorname{Eval}_{s k}(x)$; if $b=1$ then $\mathcal{A}$ is given a random $y \in R_{n}$.

4. $\mathcal{A}$ makes queries as in step 2 , as long as none of these queries is equal to $x$.

5. $\mathcal{A}$ outputs $b^{\prime}$ and succeeds if $b^{\prime}=b$ at the end of the experiment.

We require that the success probability of any PPT adversary $\mathcal{A}$ is $1 / 2+n e g l(n)$.

\section{Model}

In a typical protocol, parties are viewed as either honest or semi-honest/ malicious. To model rationality, we consider players' utilities. Here we assume that $\mathcal{F}=\{f: X \times Y \rightarrow Z\}$ is a functionality where $|X|=|Y|$ and their domain is polynomial in size $(\operatorname{poly}(n))$. Let $\mathcal{D}$ be the domain of output which is polynomial in size. The function returns a vector $I$ that represents the set-intersection where $I_{t}$ is set to one if item $t$ is in the set-intersection. In other words, for all the data items of the parties (i.e., $X$ and $Y$ ), we will compute $X \cap Y$, and we get $I$ as the output of the function. Clearly for calculating set-intersection, we need to calculate $x_{e} \wedge y_{e}$ for each $e$ where $x_{e} \in X$ and $y_{e} \in Y$. Similarly, for set-union, we need to calculate $x_{e} \vee y_{e}$ for all $e$. This can be rewritten as $\neg\left(\neg x_{e} \wedge \neg y_{e}\right)$. Computing the set-union is thus straight forward.

Given that $j$ parties are active during the computation phase, let the outcome $o$ of the computation phase be a vector of length $j$ with $o_{j}=1$ iff the output of $P_{j}$ is equal to the exact intersection (i.e., $P_{j}$ learns the correct output). Let $\nu_{j}(o)$ be the utility of player $P_{j}$ for the outcome $o$. Following [1410], we make the following assumptions about the utility functions of the players:

- If $o_{j}>o_{j}^{\prime}$, then $\nu\left(o_{j}\right)>\nu\left(o_{j}^{\prime}\right)$

- If $o_{j}=o_{j}^{\prime}$ and $\sum_{j} o_{j}<\sum_{j} o_{j}^{\prime}$, then $\nu\left(o_{j}\right)>\nu\left(o_{j}^{\prime}\right)$

In other words, player $P_{j}$ first prefers outcomes in which he learns the output; otherwise, $P_{j}$ prefers strategies in which the fewest number of other players learn the result (in our two-party case, the other player learns). From the point of view of $P_{j}$, we consider the following three cases of utilities for the outcome $o$ where $U^{*}>U>U^{\prime}$ :

- If only $P_{j}$ learns the output, then $\nu_{j}(o)=U^{*}$.

- If $P_{j}$ learns the output and the other player does also, then $\nu_{j}(o)=U$.

- If $P_{j}$ does not learn the output, then $\nu_{j}(o)=U^{\prime}$. 
So, we have the expected utility of a party who outputs a random guess for the output 2 (assuming other party aborts without any output, or with the wrong output) as follows: $U_{\text {rand }}=\frac{1}{|\mathcal{D}|} \cdot U^{*}+\left(1-\frac{1}{|\mathcal{D}|}\right) \cdot U^{\prime}$.

Also, we assume that $U>U_{\text {rand }}$; else players have almost no incentive to run the computation phase at all. As in [10, we make no distinction between outputting the wrong secret and outputting a special 'don't know' symbol- both are considered as a failure to output the correct output.

To complete the protocol, we need to provide a way for parties to identify the real iteration. Some work [1/222 29] allows parties to identify the real iteration as soon as it occurs. This approach could be used in our protocol if we assume simultaneous channels. But, this approach is vulnerable to an obvious rushing strategy when simultaneous channels are not available. To avoid this, we follow the approach shown in [10]: delay the signal indicating whether a given iteration is real or fake until the following iteration. In this case, until being sure of the occurance of real iteration, a party cannot risk aborting. Moreover, once a party learns that the real iteration occurred, the real iteration is over and all parties can compute the real output. Simultaneous channels are thus not needed in this process at the price of adding only a single round.

\section{Rational Set-Intersection Protocol}

\subsection{An Overview of the Protocol}

Let $x$ denote the input of $P_{1}$, let $y$ denote the input of $P_{2}$, and let $f$ denote the set-intersection function they are trying to compute. We follow the same highlevel approach as in 1412 29 122 23. Our intersection computation protocol proceeds in a sequence of 'fake' iterations followed by a single 'real' iteration. As in 132110, our protocol is composed of two stages, where the first stage can be viewed as a pre-processing stage and the second stage that computes the intersection takes place in a sequence of $r=r(n)$ iterations. Briefly speaking, the stages have the following form:

\section{Pre-processing stage:}

- A value $i^{*} \in\{1, \ldots, r\}$ is chosen according to some geometric distribution $0<\alpha<1$ where $\alpha$ depends on the players' utilities (discussed later in Section 5). This represents the iteration, in which parties will learn the 'true output'.

- For $i<i^{*},\left\{a_{i}\right\}=\left\{a_{1}, \ldots, a_{r}\right\}$ (resp., $\left.\left\{b_{i}\right\}=\left\{b_{1}, \ldots, b_{r}\right\}\right)$ are chosen according to some distribution that is independent of $y$ (resp., $x$ ). For $i \geq i^{*}$, $a_{i}=b_{i}=f(x, y)$.

- Each $a_{i}$ is randomly divided into shares $a_{i}^{(1)}, a_{i}^{(2)}$ with $a_{i}^{(1)} \oplus a_{i}^{(2)}=a_{i}$ (and similarly for each $b_{i}$ ). The stage concludes with $P_{1}$ being given $a_{1}^{(1)}$,

${ }^{2}$ We do not consider $U^{\prime \prime}$ - the utility when neither party learns the output, since 'not learning the output' is not the target of a rational adversary in practice. 
$b_{1}^{(1)}, \ldots, a_{r}^{(1)}, b_{r}^{(1)}$, and $P_{2}$ being given $a_{1}^{(2)}, b_{1}^{(2)}, \ldots, a_{r}^{(2)}, b_{r}^{(2)}$ alongside the

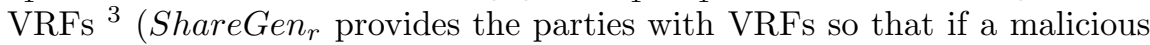
party modifies the share it sends to the other party, then the other party will almost certainly detect this due to the property of VRFs. It will be treated as an abort if such manipulation is detected.).

After this stage, each party has a set of random shares that reveal nothing about the other party's input.

\section{Intersection Computation Phase:}

In each iteration $i$, for $i=1, \ldots, r$, the parties do the following: First, $P_{2}$ sends $a_{i}^{(2)}$ to $P_{1}$ who reconstructs $a_{i}$; then $P_{1}$ sends $b_{i}^{(1)}$ to $P_{2}$ who reconstructs $b_{i}$. (Parties also checks the VRF but we omit this here.) If a party aborts in some iteration $i$, then the other party outputs the value reconstructed in the previous iteration. Otherwise, after reaching iteration $r$ the parties output $a_{r}$ and $b_{r}$, respectively. To compute the correct intersection, parties run a sequence of iterations until the real iteration is identified, and both parties output the result at that point. If some party fails to follow the protocol, the other party aborts. In fact, it is rational for $P_{j}$ to follow the protocol as long as the expected gain of deviating is positive only if $P_{j}$ aborts exactly in iteration $i^{*}$; and is outweighed by the expected loss if $P_{j}$ aborts before iteration $i^{*}$. The intersection computation phase proceeds in a series of iterations, where each iteration consists of one message sent by each party. Since we want to avoid simultaneous communication, we simply require $P_{2}$ to communicate first in each iteration.

When $X$ and $Y$ (the domains of $f$ ) are polynomial size, we follow 1321 and set $a_{i}=f(x, \hat{y})$ for $\hat{y}$ chosen uniformly from $Y$, and set $b_{i}=f(\hat{x}, y)$ for $\hat{x}$ chosen uniformly (and independently) from $X$. Note that $a_{i}$ (resp., $b_{i}$ ) is independent of $y$ (resp., $x$ ), as desired.

\subsection{Protocol Construction}

As described above, our protocol $\Pi$ consists of two stages. Let $p$ be an arbitrary polynomial, and set $r=p \cdot|Y|$. We implement the first stage of $\Pi$ using a subprotocol $\pi$ for computing a randomized functionality ShareGen (parameterized by a polynomial $r$ ) defined in Figure 1. This functionality returns shares to each party, alongside $r$-time VRF (Gen, Eval, Prove, Verify). In the second stage of $\Pi$, the parties exchange these shares in a sequence of $r$ iterations as described in Figure 2. The protocol returns $I$ at the end of the operations on all the data items.

${ }^{3}$ It is the parties' own interest that they input the correct values for ShareGen . Otherwise, they will receive incorrect shares that will give them no chance to compute the correct intersection result, which will only enable them of having smaller incentives. 
Input: Let the inputs to ShareGen $r$ be $x \in X_{n}$ and $y \in Y_{n}$. (If one of the received inputs is not in the correct domain, a default input is substituted.)

\section{Computation:}

- Define values $a_{1}, \ldots, a_{r}$ and $b_{1}, \ldots, b_{r}$ in the following way:

- Choose $i^{*}$ according to some geometric distribution $\alpha$

- For $i<i^{*}$ do,

- Choose $\hat{y} \leftarrow Y_{n}$ and set $a_{i}=f_{n}(x, \hat{y})$

- Choose $\hat{x} \leftarrow X_{n}$ and set $b_{i}=f_{n}(\hat{x}, y)$

- For $i=i^{*}$, set $a_{i}=b_{i}=q=f_{n}(x, y)$.

- For $i>i^{*}$, set $a_{i}=b_{i}=N U L L$

- For all iteration $i$, choose $\left(a_{i}^{(1)}, a_{i}^{(2)}\right)$ and $\left(b_{i}^{(1)}, b_{i}^{(2)}\right)$ as random secret shares of $a_{i}$ and $b_{i}$, respectively. (I.e., $a_{i}^{(1)} \oplus a_{i}^{(2)}=a_{i}, b_{i}^{(1)} \oplus b_{i}^{(2)}=b_{i}$ )

- Let $\mathcal{D}=\{0,1\}^{l}$ be the domain of the output. Let (Gen, Eval, Prove, Verify) and $\left(\right.$ Gen $^{\prime}$, Eval $^{\prime}$, Prove $^{\prime}$, Verif $\left.^{\prime}\right)$ be VRFs with range $\{0,1\}^{l}$ and $\{0,1\}^{n}$, respectively. Compute $\left(p k_{1}, s k_{1}\right),\left(p k_{2}, s k_{2}\right) \leftarrow \operatorname{Gen}\left(1^{n}\right)$ and $\left(p k_{1}^{\prime}, s k_{1}^{\prime}\right)$, $\left(p k_{2}^{\prime}, s k_{2}^{\prime}\right) \leftarrow \operatorname{Gen}^{\prime}\left(1^{n}\right)$. For all $i$, compute $\operatorname{share}_{i}=\operatorname{Eval}_{s k_{2}}\left(i \| b_{i}^{(1)}\right)$ and share $2_{i}=\operatorname{Eval}_{s k_{1}}\left(i \| a_{i}^{(1)}\right)$. Also compute signal1 $=\operatorname{Eval}_{s k_{2}^{\prime}}^{\prime}\left(i^{*}+1\right)$ and signal2 $=$ Eval $_{s k_{1}^{\prime}}^{\prime}\left(i^{*}+1\right)$

Output:

- Send to $P_{1}$ the values $\left(s k_{1}, s k_{1}^{\prime}, p k_{2}, p k_{2}^{\prime}, a_{1}^{(1)}, \ldots, a_{r}^{(1)},\left(b_{1}^{(1)}\right.\right.$, share $\left.1_{1}\right), \ldots,\left(b_{r}^{(1)}\right.$, share $\left.1_{r}\right)$, signal1).

- Send to $P_{2}$ the values $\left(s k_{2}, s k_{2}^{\prime}, p k_{1}, p k_{1}^{\prime}, b_{1}^{(1)}, \ldots, b_{r}^{(1)},\left(a_{1}^{(1)}\right.\right.$, share $\left.2_{1}\right), \ldots,\left(a_{r}^{(1)}\right.$, share $\left.2_{r}\right)$, signal2).

Fig. 1. Functionality ShareGen

\section{Protocol Analysis}

Here we will give some intuition as to why the reconstruction phase of $\Pi$ is a computational Nash equilibrium for an appropriate choice of $\alpha$. Let us assume that $P_{2}$ follows the protocol, and $P_{1}$ deviates from the protocol. (It is easier to analyze the deviations by $P_{2}$ since $P_{2}$ starts in every iteration.) As soon as it receives $z_{2}^{(i)}=$ signal1, $P_{1}$ can abort in iteration $i=i^{*}+1$, or it can abort in some iteration $i<i^{*}+1$. While aborting in $i=i^{*}+1, P_{1}$ 'knows' that it learned the correct output in the preceding iteration (iteration $i^{*}$ ) and can thus output the correct result; however, $P_{2}$ will output the correct result as well since it sent the $z_{2}^{(i)}=$ signal1 value to $P_{1}$. So $P_{1}$ does not increase its utility beyond what it would achieve by following the protocol. In the second case, when $P_{1}$ aborts in some iteration $i<i^{*}+1$, the best strategy $P_{1}$ can adopt is to output $a_{1}^{(i)}$ hoping that $i=i^{*}$. Thus, following this strategy, the expected utility that $P_{1}$ obtains can be calculated as follows: 
Input: Party $P_{1}$ has input $x$ and party $P_{2}$ has input $y$.

Computation:

- Preliminary phase:

1. $P_{1}$ chooses $\hat{y} \in Y_{n}$ uniformly at random, and sets $a_{0}=f_{n}(x, \hat{y})$. Similarly, $P_{2}$ chooses $\hat{x} \in X_{n}$ uniformly at random, and sets $b_{0}=f_{n}(\hat{x}, y)$.

2. Parties $P_{1}$ and $P_{2}$ run a protocol $\pi$ to compute ShareGen $r$, using their inputs $x$ and $y$.

3. If $P_{2}$ receives $\perp$ from the above computation, it outputs $b_{0}$ and halts. Otherwise, the parties proceed to the next step.

4. Denote the output of $P_{1}$ from $\pi$ by $\left(s k_{1}, s k_{1}^{\prime}, p k_{2}, p k_{2}^{\prime}, a_{1}^{(1)}, \ldots, a_{r}^{(1)}\right.$, $\left(b_{1}^{(1)}\right.$, share $\left.1_{1}\right), \ldots,\left(b_{r}^{(1)}\right.$, share $\left.1_{r}\right)$, signal 1$)$.

5. Denote the output of $P_{2}$ from $\pi$ by $\left(s k_{2}, s k_{2}^{\prime}, p k_{1}, p k_{1}^{\prime}, b_{1}^{(1)}, \ldots, b_{r}^{(1)}\right.$, $\left(a_{1}^{(1)}\right.$, share $\left.2_{1}\right), \ldots,\left(a_{r}^{(1)}\right.$, share $\left.2_{r}\right)$, signal 2$)$.

- Intersection Computation Phase

For all $i$ do:

$P_{2}$ sends message to $P_{1}$ :

1. $P_{2}$ computes $y_{2}^{(i)}=\operatorname{Prove}_{s k_{2}}\left(i \| a_{i}^{(2)}\right), z_{2}^{(i)}=\operatorname{Eval}_{s k_{2}^{\prime}}^{\prime}(i), \bar{z}_{2}^{(i)}=\operatorname{Prove}_{s k_{2}^{\prime}}^{\prime}(i)$. It sends $\left(a_{i}^{(2)}\right.$, share $\left.2_{i}, y_{2}^{(i)}, z_{2}^{(i)}, \bar{z}_{2}^{(i)}\right)$ to $P_{1}$.

2. If $P_{2}$ does not send anything to $P_{1}$, then $P_{1}$ outputs $a_{i-1}$ and halts. $P_{2}$ sends $\left(a_{i}^{(2)}\right.$, share $\left.2_{i}, y_{2}^{(i)}, z_{2}^{(i)}, \bar{z}_{2}^{(i)}\right)$ to $P_{1}$. If Verif $y_{p k_{2}}\left(i \| a_{i}^{(2)}\right.$, share $\left.2_{i}, y_{2}^{(i)}\right)=0$ or Verif $y_{p k_{2}^{\prime}}^{\prime}\left(i, z_{2}^{(i)}, \bar{z}_{2}^{(i)}\right)=0$, then $P_{1}$ outputs $a_{i-1}$ and halts. If signal $1 \neq z_{2}^{(i)}$ then $P_{1}$ outputs $a_{i-1}$, sends its iteration- $i$ message to $P_{2}$, and halts.

3. If Verif $y_{p k_{2}}\left(i \| a_{i}^{(2)}\right.$, share $\left.2_{i}, y_{2}^{(i)}\right)=1$ and $a_{i}^{(1)} \oplus a_{i}^{(2)} \neq N U L L$ (i.e., $x=x_{i}$ ), then $P_{1}$ sets $a_{i}=a_{i}^{(1)} \oplus a_{i}^{(2)}$, and continues running the protocol.

$P_{1}$ sends message to $P_{2}$ :

1. $P_{1}$ computes $y_{1}^{(i)}=\operatorname{Prove}_{s k_{1}}\left(i \| b_{i}^{(1)}\right), z_{1}^{(i)}=\operatorname{Eval}_{s k_{1}^{\prime}}^{\prime}(i), \bar{z}_{1}^{(i)}=\operatorname{Prove}_{s k_{1}^{\prime}}^{\prime}(i)$. It sends $\left(b_{i}^{(1)}\right.$, share $\left.1_{i}, y_{1}^{(i)}, z_{1}^{(i)}, \bar{z}_{1}^{(i)}\right)$ to $P_{2}$.

2. If $P_{1}$ does not send anything, then $P_{2}$ outputs $b_{i-1}$ and halts. $P_{1}$ sends $\left(b_{i}^{(1)}\right.$, share $\left.1_{i}, y_{1}^{(i)}, z_{1}^{(i)}, \bar{z}_{1}^{(i)}\right)$ to $P_{2}$. If Verif $y_{p k_{1}}\left(i \| b_{i}^{(1)}\right.$, share $\left._{i}, y_{1}^{(i)}\right)=0$ or Verif $y_{p k_{1}^{\prime}}^{\prime}\left(i, z_{1}^{(i)}, \bar{z}_{1}^{(i)}\right)=0$, then $P_{2}$ outputs $b_{i-1}$ and halts. If signal $2 \neq z_{1}^{(i)}$ then $P_{2}$ outputs $b_{i-1}$, sends its iteration- $i$ message to $P_{1}$, and halts.

3. If Verif $y_{p k_{1}}\left(i \| b_{i}^{(1)}\right.$, share $\left.1_{i}, y_{1}^{(i)}\right)=1$ and $b_{i}^{(1)} \oplus b_{i}^{(2)} \neq N U L L$ (i.e., $y=y_{i}$ ), then $P_{2}$ sets $b_{i}=b_{i}^{(1)} \oplus b_{i}^{(2)}$, and continues running the protocol.

Output: If all $r$ iterations have been run, party $P_{1}$ outputs $a_{r}$ and party $P_{2}$ outputs $b_{r}$.

Fig. 2. Protocol for computing the functionality for set-intersection

- $P_{1}$ aborts exactly in iteration $i=i^{*}$. In this case, the utility that $P_{1}$ gets is at most $U^{*}$.

- When $i<i^{*}, P_{1}$ has 'no information' about correct $a_{r}$ and so the best it can do is guess. In this case, the expected utility of $P_{1}$ is at most $U_{\text {rand }}$. 
Considering the above, $P_{1}$ 's expected utility of following this strategy is at most:

$$
\alpha \times U^{*}+(1-\alpha) \times U_{\text {rand }}
$$

Now, it is possible to set the value of $\alpha$ such that the expected utility of this strategy is strictly less than $U$, since $U_{\text {rand }}<U$ by assumption. In such a case, $P_{1}$ has no incentive to deviate. Since there is always a unique valid message a party can send and anything else is treated as an abort, it follows that the protocol $\Pi$ induces a strict computational Nash equilibrium which is stable with respect to trembles.

The detailed proof of the following propositions will be given in the full version of the paper.

Proposition 1. The protocol $\Pi$ induces a computational Nash equilibrium given that $0<\alpha<1, U>\alpha \times U^{*}+(1-\alpha) \times U_{\text {rand }}$, and the pseudorandomness of VRFs.

Proposition 2. If $0<\alpha<1, U>\alpha \times U^{*}+(1-\alpha) \times U_{\text {rand }}$, VRFs are pseudorandom, and there is always a unique valid message each party can send, then the protocol $\Pi$ induces a computational strict Nash equilibrium.

Proposition 3. The protocol $\Pi$ is stable with respect to trembles given that $0<\alpha<1$ and $U>\alpha \times U^{*}+(1-\alpha) \times U_{\text {rand }}$.

According to the above propositions and their proofs, we give the theorem as follows:

Theorem 1. If $0<\alpha<1, U>\alpha \times U^{*}+(1-\alpha) \times U_{\text {rand }}$, and VRFs are pseudorandom, then $\Pi$ induces a computational strict Nash equilibrium that is stable with respect to trembles.

\section{Conclusion}

In this paper, we have proposed a privacy-preserving set-intersection protocol in two-party settings from the game-theoretic perspective. We have used verifiable random functions as the underlying cryptographic primitive which is simple and efficient. It is also possible to use our protocol for computing set-union operations. We also show that our protocol satisfies computational versions of strict Nash equilibrium and stability with respect to trembles. Applying gametheoretic approach for multi-party setting where parties are allowed to collude is an interesting open problem.

\section{References}

1. Abraham, I., Dolev, D., Gonen, R., Halpern, J.: Distributed Computing Meets Game Theory: Robust Mechanisms for Rational Secret Sharing and Multi-party Computation. In: 25th ACM Symposium Annual on Principles of Distributed Computing, pp. 53-62 (2006) 
2. Agrawal, R., Terzi, E.: On Honesty in Sovereign Information Sharing. In: Ioannidis, Y., Scholl, M.H., Schmidt, J.W., Matthes, F., Hatzopoulos, M., Böhm, K., Kemper, A., Grust, T., Böhm, C. (eds.) EDBT 2006. LNCS, vol. 3896, pp. 240-256. Springer, Heidelberg (2006)

3. Aumann, Y., Lindell, Y.: Security Against Covert Adversaries: Efficient Protocols for Realistic Adversaries. In: Vadhan, S.P. (ed.) TCC 2007. LNCS, vol. 4392, pp. 137-156. Springer, Heidelberg (2007)

4. Bellare, M., Micali, S.: Non-interactive Oblivious Transfer and Applications. In: Brassard, G. (ed.) CRYPTO 1989. LNCS, vol. 435, pp. 547-557. Springer, Heidelberg (1990)

5. Cramer, R., Damgard, I., Nielsen, J.B.: Multi-party Computation from Threshold Homomorphic Encryption. In: Pfitzmann, B. (ed.) EUROCRYPT 2001. LNCS, vol. 2045, pp. 280-299. Springer, Heidelberg (2001)

6. Dodis, Y.: Efficient Construction of (distributed) Verifiable Random Functions. In: Desmedt, Y.G. (ed.) PKC 2003. LNCS, vol. 2567, pp. 1-17. Springer, Heidelberg (2002)

7. Dodis, Y., Rabin, T.: Cryptography and Game Theory. In: Nisan, N., Roughgarden, T., Tardos, E., Vazirani, V. (eds.) Algorithmic Game Theory, pp. 181-207. Cambridge University Press, Cambridge (2007)

8. Dodis, Y., Yampolskiy, A.: A Verifiable Random Function with Short Proofs and Keys. In: Vaudenay, S. (ed.) PKC 2005. LNCS, vol. 3386, pp. 416-431. Springer, Heidelberg (2005)

9. Emura, K., Miyaji, A., Rahman, M.S.: Efficient Privacy-Preserving Data Mining in Malicious Model. In: Cao, L., Feng, Y., Zhong, J. (eds.) ADMA 2010, Part I. LNCS, vol. 6440, pp. 370-382. Springer, Heidelberg (2010)

10. Fuchsbauer, G., Katz, J., Naccache, D.: Efficient Rational Secret Sharing in Standard Communication Networks. In: Micciancio, D. (ed.) TCC 2010. LNCS, vol. 5978, pp. 419-436. Springer, Heidelberg (2010)

11. Goldreich, O.: Foundations of cryptography: Basic applications. Cambridge Univ. Press, Cambridge (2004)

12. Gordon, S.D., Katz, J.: Rational Secret Sharing, Revisited. In: De Prisco, R., Yung, M. (eds.) SCN 2006. LNCS, vol. 4116, pp. 229-241. Springer, Heidelberg (2006)

13. Gordon, S.D., Hazay, C., Katz, J., Lindell, Y.: Complete Fairness in Secure Twoparty Computation. In: 40th Annual ACM Symposium on Theory of Computing, STOC 2008, pp. 413-422 (2008)

14. Halpern, J., Teague, V.: Rational Secret Sharing and Multi-party Computation: Extended abstract. In: 36th Annual ACM Symposium on Theory of Computing, STOC 2004, pp. 623-632 (2004)

15. Izmalkov, S., Micali, S., Lepinski, M.: Rational Secure Computation and Ideal Mechanism Design. In: 46th Annual Symposium on Foundations of Computer Science, FOCS 2005, pp. 585-595 (2005)

16. Jagannathan, G., Wright, R.N.: Privacy-preserving Distributed k-means Clustering over Arbitrarily Partitioned Data. In: ACM SIGKDD International Conference on Knowledge Discovery and Data Mining, KDD 2005, pp. 593-599 (2005)

17. Jiang, W., Clifton, C., Kantarcioglu, M.: Transforming Semi-Honest Protocols to Ensure Accountability. Data and Knowledge Engineering (DKE) 65(1), 57-74 (2008)

18. Kantarcioglu, M., Clifton, C.: Privately Computing a Distributed k-nn Classifier. In: Boulicaut, J.-F., Esposito, F., Giannotti, F., Pedreschi, D. (eds.) PKDD 2004. LNCS (LNAI), vol. 3202, pp. 279-290. Springer, Heidelberg (2004) 
19. Kantarcioglu, M., Kardes, O.: Privacy-preserving Data Mining in the Malicious model. International Journal of Information and Computer Security 2(4), 353-375 (2008)

20. Katz, J.: Bridging Game Theory and Cryptography: Recent Results and Future Directions. In: Canetti, R. (ed.) TCC 2008. LNCS, vol. 4948, pp. 251-272. Springer, Heidelberg (2008)

21. Katz, J.: On Achieving the Best of Both Worlds in Secure Multi-party Computation. In: 39th Annual ACM Symposium on Theory of Computing, STOC 2007, pp. $11-20(2007)$

22. Kol, G., Naor, M.: Cryptography and Game Theory: Designing Protocols for Exchanging Information. In: Canetti, R. (ed.) TCC 2008. LNCS, vol. 4948, pp. 320 339. Springer, Heidelberg (2008)

23. Kol, G., Naor, M.: Games for Exchanging Information. In: 40th Annual ACM Symposium on Theory of Computing, STOC 2008, pp. 423-432 (2008)

24. Lin, X., Clifton, C., Zhu, M.: Privacy-preserving Clustering with Distributed EM Mixture Modeling. Knowledge and Information Systems 8(1), 68-81 (2005)

25. Lindell, Y., Pinkas, B.: Privacy-preserving Data Mining. In: Bellare, M. (ed.) CRYPTO 2000. LNCS, vol. 1880, pp. 36-54. Springer, Heidelberg (2000)

26. Lipmaa, H.: Verifiable Homomorphic Oblivious Transfer and Private Equality Test. In: Laih, C.-S. (ed.) ASIACRYPT 2003. LNCS, vol. 2894, pp. 416-433. Springer, Heidelberg (2003)

27. Liu, J., Lu, Y.H., Koh, C.K.: Performance Analysis of Arithmetic Operations in Homomorphic Encryption. In: ECE Technical Reports, Purdue University (2010)

28. Lysyanskaya, A.: Unique Signatures and Verifiable Random Functions from the DH-DDH Separation. In: Yung, M. (ed.) CRYPTO 2002. LNCS, vol. 2442, pp. 597-612. Springer, Heidelberg (2002)

29. Lysyanskaya, A., Triandopoulos, N.: Rationality and Adversarial behavior in Multiparty computation. In: Dwork, C. (ed.) CRYPTO 2006. LNCS, vol. 4117, pp. 180 197. Springer, Heidelberg (2006)

30. Micali, S., Rabin, M.O., Vadhan, S.P.: Verifiable Random Functions. In: 40th Annual Symposium on Foundations of Computer Science, FOCS 1999, pp. 120-130 (1999)

31. Miyaji, A., Rahman, M.S.: Privacy-preserving Data Mining in Presence of Covert Adversaries. In: Cao, L., Feng, Y., Zhong, J. (eds.) ADMA 2010, Part I. LNCS, vol. 6440, pp. 429-440. Springer, Heidelberg (2010)

32. Nix, R., Kantarcioglu, M.: Incentive Compatible Distributed Data Mining. In: IEEE International Conference on Privacy, Security, Risk and Trust, pp. 735-742 (2010)

33. Ong, S.J., Parkes, D., Rosen, A., Vadhan, S.: Fairness with an Honest Minority and a Rational Majority. In: Reingold, O. (ed.) TCC 2009. LNCS, vol. 5444, pp. 36-53. Springer, Heidelberg (2009)

34. Su, C., Bao, F., Zhou, J., Takagi, T., Sakurai, K.: Security and Correctness Analysis on Privacy-Preserving k-Means Clustering Schemes. IEICE Trans. Fundamentals E92-A(4), 1246-1250 (2009)

35. Vaidya, J., Clifton, C.: Privacy Preserving Association Rule Mining in Vertically Partitioned Data. In: ACM SIGKDD International Conference on Knowledge Discovery and Data Mining, KDD 2002, pp. 639-644 (2002)

36. Zhang, N., Zhao, W.: Distributed Privacy-preserving Information Sharing. In: The 31st International Conference on Very Large Data Bases, VLDB 2005, pp. 889-900 (2005) 